\title{
A Hyper-Heuristic Heterogeneous Multisensor Node Scheme for Energy Efficiency in Larger Wireless Sensor Networks Using DEEC-Gaussian Algorithm
}

\author{
Oluwasegun Julius Aroba $(\mathbb{D}$, Nalindren Naicker $(\mathbb{D}$, and Timothy Adeliyi \\ ICT and Society Research Group, Information Systems, Durban University of Technology, 4001 Durban, South Africa \\ Correspondence should be addressed to Nalindren Naicker; nalindrenn@dut.ac.za
}

Received 17 December 2020; Revised 26 January 2021; Accepted 3 February 2021; Published 15 February 2021

Academic Editor: Jose M. Barcelo-Ordinas

Copyright (C) 2021 Oluwasegun Julius Aroba et al. This is an open access article distributed under the Creative Commons Attribution License, which permits unrestricted use, distribution, and reproduction in any medium, provided the original work is properly cited.

\begin{abstract}
A wireless sensor network (WSN) is an intellect-sustainable network that comprises multiple spatially distributed sensor nodes and several sink nodes that collect data from sensors. WSNs remain an active research area in the literature due to challenging factors such as the selection of sensor location according to a given premise, finding optimal routing algorithm, and ensuring energy efficiency and consumption. Minimizing energy and prolonging the network lifetime in the WSNs are the focus of this research work. In the literature, a clustering approach is used in grouping sensor nodes into clusters and is seen as an effective technique used in optimizing energy consumption in WSNs. Hence, in this paper, we put forward a novel clustering-based approach by amalgamating the Gaussian elimination method with the Distributed Energy-Efficient Clustering to produce DEEC_Gaussian (DEEC_Gaus) to stabilize energy efficiency optimization in WSNs. We took the advantages of DEEC and Gaussian elimination algorithms to resolve energy efficiency problems in WSNs. DEEC presents attributes such as increased heterogeneity performance level, clustering stability in operation, and energy efficiency which helps to prolong network lifetime while the Gaussian elimination algorithm added an additional advantage to improve and optimize energy efficiency, to aggregate packets of operations performed in the network lifestyle of energy efficiency in WSNs. The simulations were carried out using MATLAB software with 1000 to 1500 nodes. The performance of the proposed work was compared with state-of-the-art algorithms such as DEEC, DDEEC, and EDEEC_E. The simulated results presented show that the proposed DEEC-Gauss outperformed the three other conventional algorithms in terms of network lifetime, first node dead, tenth node dead, alive nodes, and the overall timing of the packets received at the base station. The results showed that the proposed hyper-heuristic heterogeneous multisensor DEEC-Gauss algorithm presented an average percentage of 3.0\% improvement for the tenth node dead (TND) and further improvement of $4.8 \%$ for the first node dead (FND). When the performance was compared to the state-of-the-art algorithms in larger networks, the overall delivery was greatly improved and optimized.
\end{abstract}

\section{Introduction}

Wireless sensor networks (WSNs) are networks that comprise multihop communication systems that are a bunch of battery-powered sensor nodes that are used to effectively monitor the processing unit and storage unit for collating and analyzing information with the use of their sensor modules [1-4]. WSN applications are used in different sectors such as the health care sector for surveillance, rescue surveillance, environmental monitoring and disaster recovery, security, coverage, location identification services, target tracking systems, and battlefield surveillance [5]. Usually, most Internet of Things (IoT) and applications of sensors applications are deployed randomly without their locations being known. On the other hand, some applications make use of sensor nodes in multinumbers [6]. In the literature, an improved node localization accuracy with energy efficiency gave birth to future recommendations on the trade-off between localization and energy accuracy [7]. WSNs pointed out a distinctive area to monitor application for identifying the unknown sensor node and anchor nodes. Localization is utilized to 
prove the exact location of indoor and outdoor environments, namely, universities, hospitals, health care organization centers, and shopping complexes [8]. DEEC is an energy-efficient protocol for homogeneous performances, and it is not well suitable for heterogeneous operations in WSNs for energy efficiency. However, an electricity-saving scheme is deployed for homogeneous WSN that is not appropriate in deployment [9].

There are different categories of routing techniques in WSNs which are performed with the different points of views used for efficient usage of a few sensor nodes energy where the cluster for every cluster head $(\mathrm{CH})$ nominates most of the performance in communication task. Also, the nodes for heterogeneous networks have different capabilities for choosing the high energy nodes, high data refining, and computational power as the $\mathrm{CH}$ which are classified as dynamic cluster head and static cluster head [10]. The cluster head selection processes in WSNs help manage the lifespan of the network where the $\mathrm{CH}$ conducts the data aggregation from various cluster members to be transferred to the database station (BS) for analysis [10-12].

Wireless sensor network routing has a major impact on the network lifetime to enhance the sensor networks. Routing helps to select the appropriate energy efficiency and the path to send the data from the source to the base station. The sensor nodes are categorized as source nodes and sink nodes with the medium of intermediate nodes in the network. WSNs are classified as homogeneous or heterogeneous according to their energy emission levels of sensor nodes. When all the nodes have an equal amount of energy utilization, it is said to be homogeneous while heterogeneous networks have more initial energy supplied to a few sensor nodes in comparison to the usual nodes $[13,14]$.

Literature abounds with research works on state-of-theart algorithms, namely, Distributed Energy-Efficient Clustering Extended (DEEC_E), Developed Distributed Energy-Efficient Clustering Extends (DDEEC_E), and Distributed Energy-Efficient Clustering (DEEC) with normal, advance, and supernode classifications. This research study addresses a gap in this research area as it produced the results for larger wireless sensor networks (WSNs) of over 1000 nodes, namely, 1000, 1100, 1200, 1300, 1400 , and 1500 sensor nodes for various initial energy levels, namely, $0.5,0.6,0.7$, and 0.8 joules of energy that has not been seen before using the novel DEEC_Gausian algorithm.

The main contribution of this research work is the following:

(i) To provide an effective energy efficiency optimization

(ii) To carry out an extensive simulation of the proposed heterogeneous DEEC-Gauss algorithm in MATLAB

(iii) To evaluate and compare the proposed algorithm with classical state-of-the-art algorithms using popular performance criteria: network lifetime of the algorithm, tenth node dead, alive node, and the overall timing of the packet received at the base station metrics
The rest of the paper is presented as follows: Section 2 sheds more light on the related works. Section 3 discusses the materials and methods in detail. The simulation and presentation of results for the preferred algorithm are presented in Section 4. Finally, the conclusions are presented in Section 5.

\section{Related Works}

Hyper-heuristic research consists of different approaches that distribute the common goal of automating the adaptation and design of heuristic methods to solve hard computational search issues. According to Meng et al. in [15], the 3D localization method of unmanned aerial vehicles (UAVs) is used to help to prove the sensor node localization to locate the GPS-equipped UAV [15], alleviate path interference, and reduce error and optimization for neural network-based machine learning algorithms. The outcome was able to improve the positioning accuracy and coverage while the static position was able to encounter a non-line of sight (NLoS), and their method was suitable for situations on ground anchors in an optimal position. Localization processes have two different types which are outdoor and indoor tools for global positioning systems (GPSs) [8]. According to Prakash et al. in [9], the approach of exterior bound allotted advanced node distribution (EBAN) and DEEC protocols in WSNs was explained, and computational heterogeneity, link heterogeneity, and energy heterogeneity were utilized with energy, $m=0.15,50 \%$ higher than other sensor nodes that enhanced the different transmission between cluster head $(\mathrm{CH})$ and sink node.

In the same way, the delivery of hierarchical clustering set of rules for the WSNs is considered to be the Low Energy Adaptive Clustering Hierarchy (LEACH), and DEEC is assumed to be the $\mathrm{CH}$ according to the protocols that rotate the position to slightly distribute the energy load for sensors within the network [16]. PEGASIS is a series of protocols that tend to avoid clustering formation and prefer to use the simplest one node during transmission to the base station (BS) instead of the use of a couple of nodes [17]. In a like manner, the DEEC scheme is used essentially for threedegree heterogeneity which shows that it is better than Stable Election Protocols (SEPs) that use stochastic scheme detection for extension of the community lifetime [18].

Poluru et al. in [19] tried using Energy Adaptive Distributed Energy-Efficient Clustering (EADEEC) for network stability and a lifetime of the sensor nodes in WSNs, and the further recommendation was for longevity and time of operational performance. Nehra et al. in [20] suggested Improved Distributed Energy-Efficient Clustering (I-DEEC) by distributing network nodes between two layers of normal and advanced hexagons for considering the distance between the base station within the same area. The sum of the ratio of the distance to the nodes and residual energy is calculated with the possibility of sensor nodes to nominate as the cluster head for revamps of DEEC protocols within the network lifetime, throughput, and the percentage area coverage. 
According to Priyadarshini and Sivakumar in [21], the amount of coverage by the deployment of the percentage of the enclosed circulating sensor node coverage with their application to various domains like high density areas, underwater, and forests implies the sensor node field-deployed energy dissipation according to the task assigned for network lifetime and sensing coverage area. Another solution proposed by Zhao et al. in [22] was to deploy a set of high energy frequency to aid the sensor nodes to the top for forming a block layer to remove the energy gap problem and the techniques was employed in WSNs according to the immune cloned selection of the power control. Nevertheless, some group of researchers from the literature tried to achieve the implementation of the first and second parameters on a multiheterogeneous network area to improve the lifetime of the network further. Similarly, to get the desired result for energy efficiency, some constraints of WSNs strategies were adopted for minimizing the data among the communicating parameters [23-27].

Indeed, it is likely modified that when the start point station changes location and the energy consumption of the network is at optimum, it shortens the lifetime of the network. This is why there was gateway node introduction at the center of the network [28]. Consequently, the DDEEC was compared to Improved Extended Distributed Energy Efficiency Clustering (iE-DEEC) by taking into consideration the average distance, network lifetime, and residual energy of sensor nodes and the cluster head. The performance of iEDEEC shows great improvement with the introduction of different energy amplification to reduce the energy consumption during the communication between the base station (BS) and cluster head (CH) intraoperation [29].

However, according to Kaur and Sharma in [30], Distributed Energy-Efficient Clustering protocols are used to speed its efficiency which is not vivid with the support of LEACH to conserve energy during the process of transmission of data. The DEEC is used for selecting a multicluster head at one round instead of using a single $\mathrm{CH}$ per round to increase the rate at which packets are transferred to the base station. In a like manner, Dhiman et al. in [31] presented a radical study of energy efficiency hierarchical cluster-based routing protocols for WSNs to analyze and design routing protocols on the performance of tactics when compared to the shifting paradigm. In a heterogeneous network, sensor nodes have optional energy levels to increase the EDEEC that uses three types of sensor nodes (normal, advanced, and supernodes) as supernodes have more chances to be the $\mathrm{CH}$ at the first round of transmission [32].

There have been several factors for clustering which are cluster count, selection of cluster head size, and density. The review of existing clustering algorithms on nonprobabilistic and probabilistic factors helps the mode of $\mathrm{CH}$ to $\mathrm{BS}$ communicate in both direct and indirect routing. The fault type was able to deduce that the variation of clustering approaches depends on the type of application and their usage scenarios [33]. In [34-40], more light was shed on how to save energy and how to elongate network lifetime which are the constraints for modeling of clusters. Uniformly, the technological design method with the aid of clustering algorithm deployment for independent heterogeneous WSNs was selected by Purkar and Deshpande [41]. Looking at index modeling with various node attributes, the energy efficiency was improved and node lifetime was extended.

A further recommendation was made for future work to extend the design toward the base station with the application of a genetic algorithm and cuckoo filter approach for speedy efficiency. In like manner, Rahiminasab et al. in [42] considered the cluster splitting process (CSP) method and an analytic order hierarchy process (AHP) approach to suggest an alternative solution to clustering lapses by extending the network lifetime for higher optimization compared to the base station high-powered clustering protocols, and it aids the reduction of almost 5\% than the base station controlled zestful clustering protocol algorithms. Furthermore, the recommendation is said to look into distance management, subway stations, and the control system needed, for future work [43].

Similarly, Particle Swarm Optimization (PSO) based energy systematic coverage control approach for WSNs was proposed by Ju et al. in [44]. To make the most of the data generation rate, routing protocols on the data collation controlling technology for reusable WSNs [45] and localization base evolution routing (LOBER) for an optimum collation of information in wireless multisensor networks [46] were proposed. In the same way, [47] discussed the approach of an enhanced energy-efficient forum in sensor information systems (EPEGASIS) algorithm to lift the major occurrences from various aspects, which are direct communication distance, threshold value, and mobile sink technology to adjust their communication ranges. Likewise, [48] deployed a self-sufficient Hamilton loop data for aggregating processes with moving agents for WSNs to settle and give resources back on top of the total network with the use of the PEGASIS method and the Hamilton circle.

Alternatively, the approach of maximum coverage with a multipath scheduling approach with many mobile sensor sink nodes for WSNs was proposed by [49] to help sensors to transmit and monitor data from source to sink in multicommunication means. A proposed distributed efficient algorithm for self-protection of WSNs was the distributed learning automation based algorithm as discussed in the literature [50]. Furthermore, an algorithm for the connected p-percent coverage problem in WSNs for improving network life is proposed. The innovative hyper-heuristic, Gaussian Clustering scheme for energy-efficient optimization in WSNs was the genesis of this research, and the future recommendation was to use a larger number of sensor nodes for improvement. Percent degree constraint dominating set (pDCDS) method was employed and it identifies the base number of sensor nodes for monitoring area coverage and network lifetime [51]. In brief, a distributed efficient algorithm was employed to individually safeguard WSNs in terms of efficiency and self-protection [51].

\section{Materials and Methods}

3.1. The Proposed DEEC-Gauss Network Model. This section describes the proposed DEEC-Gaussian algorithm model 
that converges the clustering-based model with separation between the sensor and cluster heads. The requirement parameters used for the proposed DEEC-Gauss are as follows. We assume the Popt $=0.1$ (i.e., it is the parameter for period mode). The nodes are randomly deployed from a base station. Most of the nodes are homogeneous and have few energies starting from $0.5 \mathrm{~J}$ to $0.8 \mathrm{~J}$. The base station is stationary at the initial point which helps to identify the location inside or outside the sensing coverage area. Every sensor node comes together and composes the data periodically and always has some data to forward. The sensor nodes were unable to identify their pinpoint positions nor the location of other sensor nodes. The nodes are selforganizing and need not be monitored after deployment.

Every node can operate as a cluster head, and the nodes are distributed uniformly and randomly to determine the lifetime of each node to know the expected energy after each round of sensor nodes. CHs are placed in the middle of the sensing area. There is a reduction of the number of nodes from the nodes to the destination which is the tracking path of the packet. Before the destination, packets from the sensor nodes must travel through the cluster head. The location of the sensor nodes is unknown to the cluster head. The WSN scenario considered for simulation has all the above criteria and disadvantages. Sensor nodes can compute the distance from the base station and other nodes by comparing the acknowledged signal. Hence, it does not need any additional system with location services such as GPS. Also, a node joins the cluster whose cluster head is the nearest to it.

3.2. Proposed Heterogeneous DEEC-Gauss Algorithm. The energy model locates the energy when the sensor sends or acknowledges data within the network. In this paper, we adopted DEEC state-of-the-art algorithm and improved it with the use of a clustering-based algorithm called Gaussian elimination with its characteristic formulation. The proposed DEEC-Gaussian algorithm seeks to administer the suggested solution as follows using the pseudocode process below:

Step 1. Set the criterion parameters.

Step 2. Start energy initialization for the sensor nodes [52], with

$$
E_{\text {Total }}=\sum_{i=1}^{n} E_{0}\left(1+a_{i}\right)=E_{0}\left(n+\sum_{i=1}^{n} a_{i}\right) .
$$

Step 3. Start iteration of the rounds as follows:

(a) Check if any dead node exists and note the round of the first node died

(b) Check if $10 \%$ of the nodes are dead and save the round that occurs

(c) Check if all the nodes are dead and save the round that occurs

(d) Check each node if alive and label it as " $N$ "

(e) Save the number of dead nodes, alive nodes, and cluster heads which are initially zero in every round (f) Iterate through each node

(i) Calculate the $p_{i}$ for heterogeneous nodes using [53]

$$
p_{i}=\frac{p_{\mathrm{opt}} N(1+a) E_{i}(r)}{\left(N+\sum_{i=1}^{N} a_{i}\right) \bar{E}(r)} .
$$

(g) Compute the energy needed by the transmitted amplifier [53]

$$
E_{\mathrm{TX}}(l, d)=\left\{\begin{array}{cc}
l E_{\mathrm{elec}}+l \varepsilon_{f_{s}} d^{2}, & d<d_{0}, \\
l E_{\mathrm{elec}}+l \varepsilon_{m p} d^{4}, & d \geq d_{0},
\end{array}\right\}
$$

and calculate the energy needed by the receiver [54]

$$
E_{R X}(l) \text { using } E_{\mathrm{RX}}(l)=E_{\text {elec }} \text {. }
$$

(h) Calculate the criteria for the sensor node energy in the step to follow [54], using

$$
E_{\text {Total }}=\sum_{i=1}^{n} E_{0}\left(1+a_{i}\right)=E_{0}\left(n+\sum_{i=1}^{n} a_{i}\right)
$$

Let $n i$ symbolize the number of steps that are to be the cluster head for the node $s i$, and we reference it as the rotating epoch, supernode, and normal node. In DEEC protocol, $E_{i}$ is used for the energy dissipated in the network. In our scheme, $E_{i}$ is used to denote the energy residual $E_{i}$ of node si for round $r$. When the cluster heads have been selected using DEEC, the selected cluster heads are now subjected to the Gaussian elimination algorithm.

Let us assume that the criterion for selected $\mathrm{CH}$ is q; matrix $A$ represents the power usage of individual nodes chosen as $\mathrm{CH}$ and $q$ the number of cluster heads. Matrix aij represents the power usage of a cluster head $i$ which is taken to be a normal node if cluster head $j$ is its cluster head. Furthermore, $b_{i}$ symbolizes the residual power source of cluster head $i$, while $x i$ expresses the times in which cluster head $i$ can equate a $\mathrm{CH}$. In this way, matrices $B$ and $X$ are formed, according to Gharib et al. [54] so that $A \cdot X=B$, as shown in

$$
\left[\begin{array}{ccccc}
a_{11} & a_{12} & a_{13} & & a_{1 k} \\
a_{21} & a_{22} & a_{23} & \cdots & a_{2 k} \\
a_{31} & a_{32} & a_{33} & & a_{3 k} \\
& \vdots & & \ddots & \vdots \\
a_{k 1} & a_{k 2} & a_{k 3} & \cdots & a_{k k}
\end{array}\right]\left[\begin{array}{c}
x_{1} \\
x_{2} \\
x_{3} \\
\vdots \\
x_{k}
\end{array}\right]=\left[\begin{array}{c}
b_{1} \\
b_{2} \\
b_{3} \\
\vdots \\
b_{k}
\end{array}\right] .
$$

The snippet of code is used to calculate the number of rounds within the entire network and to obtain the optimal summary of clusters [54].

$$
\begin{aligned}
& \text { For }(k=1 ; k<m+1 ; k++) \\
& I \_\max :=\operatorname{argmax}(i=k, \ldots, m, \operatorname{abs}(A[i, k])) ; \\
& \text { If }\left(A\left[i \_\max , k\right]=0\right)
\end{aligned}
$$




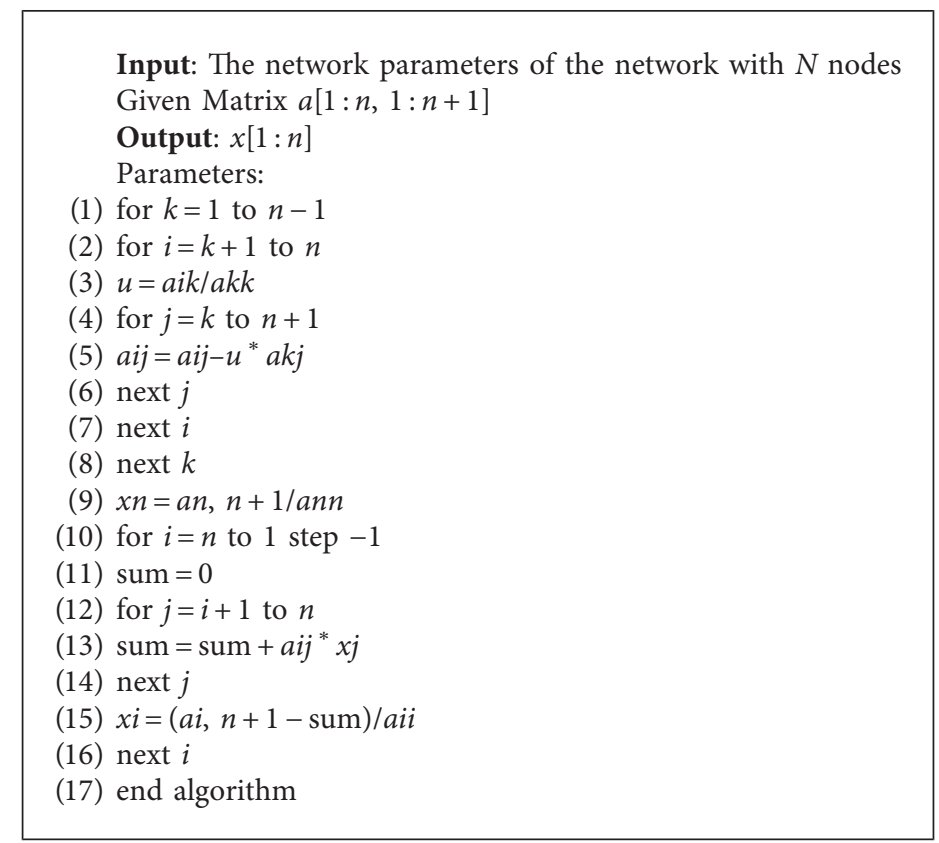

Algorithm 1: Sequential algorithm 1: Gauss elimination algorithm [54].

Error "Matrix is singular!";

Swap rows $\left(k, i \_\max \right)$;

The snippet of code is used to calculate the packet information that is sent to the BS and the tenth node dead $[54,55]$.

$$
\begin{aligned}
& \text { For }(i=k+1 ; i<m+1 ; i++) \\
& \text { For }(j=k+1 ; k<n+1 ; j++) \\
& A[i, j]:=A[i, j]-A[k, j] \times(A[i, k] / A[k, k]) ; \\
& A[i, k]:=0 ;
\end{aligned}
$$

Figure 1 represents the system model which shows the flow of the proposed DEEC-Gaussian algorithm.

3.3. Energy Consumption. The energy usage varies from $0.5 \mathrm{~J}$ to $0.8 \mathrm{~J}$ initiated at every point for the sensors by clustering nodes of the sensors in the network. The proposed approach with the use of the DEEC-Gaussian algorithm helps to provide an adaptive efficient use of energy resources of sensor nodes.

3.4. Performance Evaluation. The proposed DEEC-GAUSS was simulated in MATLAB 2020b, and the results were plotted. The system configuration was Intel Core i78650U CPU @1.90 GHz, 2.11 GHZ, installed memory (RAM) 8,00 GB (7,85 GB usable), System type 64-bit operating system, and the x64-based processor running Windows Operating System 10. The simulation was performed over 120 times with varying conditions such as identification of numbers for the nodes and the identification of cluster heads. The network sensing area was pivoted to $100 \times 100 \mathrm{~m}^{2}$. The simulations are on WSNs \#1 for 1000 to 1500 nodes and 10 cluster heads. The base station is situated at the edge of the sensor area at $(100 \times 100)$. The DEEC-GAUSS, DEEC-E, DDEEC_E, and DEEC were run 120 times, and the average of the result of the output data was selected for representing the results in the graph charts. The DEEC-Gauss algorithm was tested with a predefined clustering population. The different parameters presented for the simulation are displayed in Table 1.

3.5. Network Requirement for Deployment. The segments of the simulation in square field location are expanded by $m \times m$ meters, where $m=100$. The starting point is present at the center of the area of the location. The entire network comprises $n=1000$ to 1500 sensor nodes just as shown explicitly enough in Table 1 .

3.6. The Performance Criteria Used. The metric of parameters that are used for the performance and summarize the clustering operation lifetime, the number of sensor nodes, alive nodes, and the number of information packets acknowledged at the base station are as follows:

(i) Data Packet. It is the total number of data packets of messages that are acknowledged at the base station and their output varies from time to time.

(ii) Alive Nodes. The set of nodes that has not used up their energy.

(iii) Sensor Nodes (SNs). These are the electronic component which helps to identify the environmental parameters such as light, humidity, pressure, temperature, processing power, memory, and energy. 


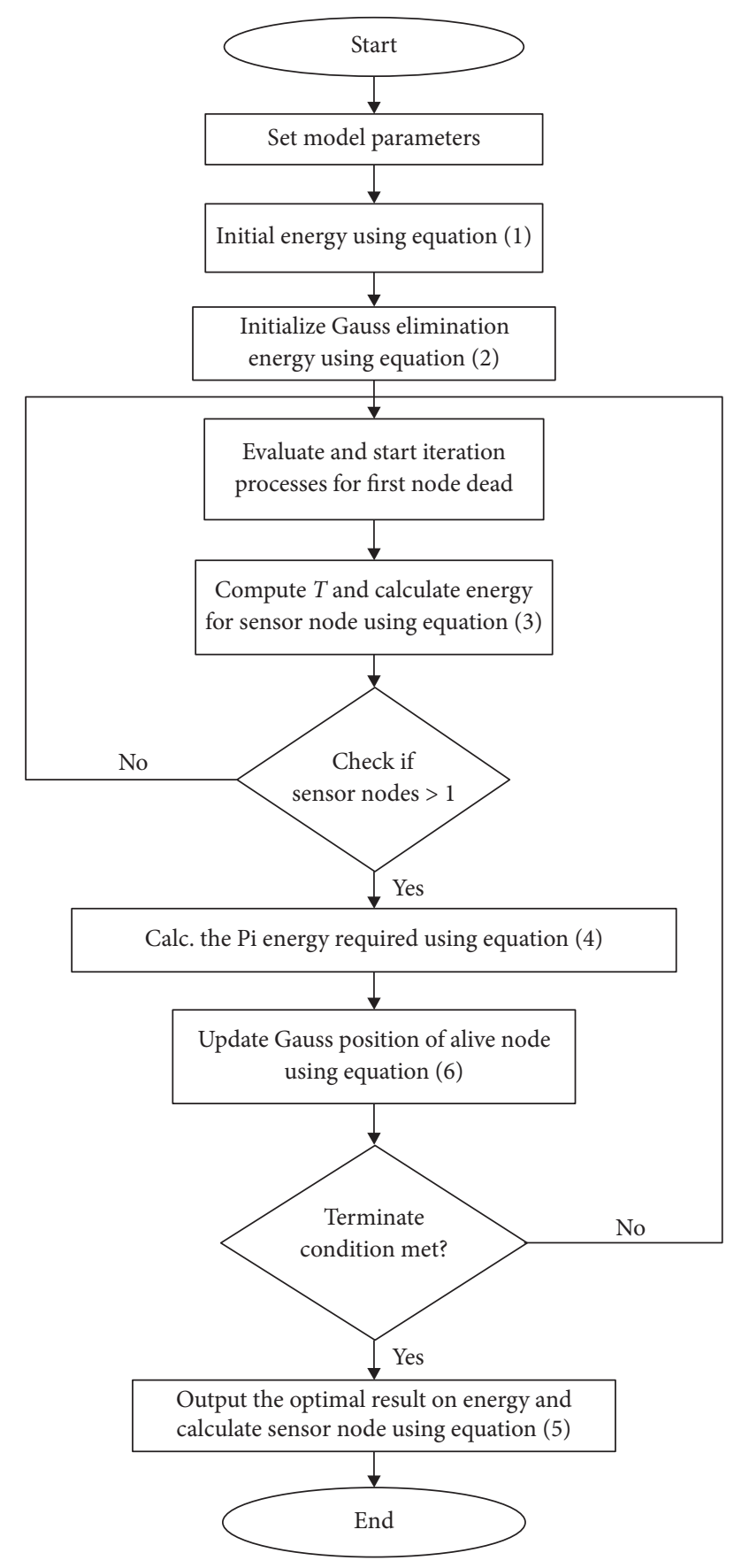

Figure 1: Proposed DEEC-Gaussian algorithm.

TABLE 1: Network simulation requirements.

\begin{tabular}{lc}
\hline Parameters & Value \\
\hline Network field & $(100,100) \mathrm{m}^{2}$ \\
Number of nodes & $1000-1500$ \\
$E_{0}$ (initial energy of normal nodes) & $0.5 \mathrm{~J}-0.8 \mathrm{~J}$ \\
Message size & $5000 \mathrm{bits}$ \\
$E_{\text {elec }}$ & $50 \mathrm{~nJ} / \mathrm{bit}$ \\
$E_{\mathrm{fs}}$ & $10 \mathrm{~nJ} / \mathrm{bit} / \mathrm{m}^{2}$ \\
$E_{\mathrm{amp}}$ & $0.0013 \mathrm{pJ} / \mathrm{bit} / \mathrm{m}^{4}$ \\
$E_{\mathrm{DA}}$ & $5 \mathrm{Nj} / \mathrm{bit} / \mathrm{signal}$ \\
$D_{0}($ threshold distance) & $100 \mathrm{~m}$ \\
Popt & 0.1 \\
\hline
\end{tabular}

(iv) Base Station (BS). This comprises more computational power, energy resources, and communication channel.

(v) Clustering. The wireless sensor network nodes are arranged in the circular form of the cluster within the sensing area range. Many nodes are deployed in the environment with its application which is classified as hierarchical, grid base scheme, and heuristic form. Clustering normally adjusts using sensing range, and metrics are used for distance measurements between the nearest node from the base station to the destination sensor node.

(vi) Cluster Head. $\mathrm{CH}$ helps to gather the packets of information from the sensors and coveys captured data to the base station.

(vii) Stability Period. It helps to account for the time taken from the primary network deployment through the path until the death of the first sensor node is thrown up.

(viii) Network Lifetime. The energy consumption in the network lifetime is identified and specified as the amount of time it takes to deplete till the death of the first node or till the death of the lower number of sensor nodes. Our purpose of such explanation is to utilize the number of sensor nodes that can represent a speculated area for a specific time.

(ix) Throughput. This is defined as the number of processes absorbed per unit time comparatively, which may be explained as the number of data sent to the cluster and collation of packets sent to the base station of the successful operation of the task.

(x) Total Residual Energy. This is defined as the points to the total of energy left in the entire network regardless of the node type. The summation of energy that is not utilized within the network per unit time represents the amount of energy taken to perform the available setup for a specific period of rounds.

\section{Simulation and Analysis}

In this section, we will throw light on the simulation results of the proposed novel DEEC-Gauss algorithm, and the comparison analysis with the state-of-the-art clustering algorithms. The state-of-the-art algorithms were Distributed Energy-Efficient Clustering Extended (DEEC_E), Developed Distributed Energy-Efficient Clustering Extends (DDEEC_E), and Distributed Energy-Efficient Clustering (DEEC) with normal, advance, and supernode classifications. These various algorithms were run at different intervals more than 360 times. Each algorithm was run 120 times using initial energy of $0.5 \mathrm{~J}$ to $0.8 \mathrm{~J}$, and the average result was selected.

4.1. Results for First Node Dead and Tenth Node Dead. The clustering algorithms were calculated with respect to the stability of the networks for the first node dead (FND), the 
number of rounds in the entire network until all the sensor nodes die and their energy gets depreciated, and lastly, the tenth node dead (TND). The number of rounds was fixed at 5,000 all through the simulation rounds with energy from $0.5 \mathrm{~J}$ to $0.8 \mathrm{~J}$. In this simulation experiment, the network lifetime was computed in terms of rounds when the first node dead for a different number of sensor nodes ranges from 1000 to 1500 sensors.

The first node dead is a crucial metric for many software applications as the feedback from the sensor node network depends on prolonging the time differences before the death of the first sensor node. Figures 2-5 show the performance of clustering algorithms, namely, DEEC_E, DDEEC_E, DEEC, and DEEC-Gauss through the simulation rounds using initial energy of $0.5 \mathrm{~J}, 0.6 \mathrm{~J}, 0.7 \mathrm{~J}$, and $0.8 \mathrm{~J}$ for First Node Dead.

4.1.1. First Node Dead. In Figure 2, it is clear that the DEECGauss for first node dead (FND) at 0.5 J initial energy was the highest from 1000 to 1500 nodes (i.e., 1668, 1653, 1621, 1563, 1588 , and 1412, respectively) while DEEC for 1000-1500 sensor nodes has the second highest (i.e., 936, 1026, 970, 979, 1036, and 1060, respectively). Figure 2 shows that the DEECGauss algorithm outclasses other state-of-the-art algorithms.

Figure 3 presents the results of the first node dead (FND) at $0.6 \mathrm{~J}$. It is clear that DEEC-Gauss is more stable and has the highest number of nodes before the death of the first sensor node, DEEC_E as second while the DEEC is the third, and lastly, the DDEEC_E presented the lowest number of sensor nodes.

Figure 5 presents the results of the first node dead (FND) at $0.7 \mathrm{~J}$ initial energy. It is clear that DEEC-Gauss surpasses with the highest number of nodes before the death of the first sensor node, DEEC_E as second while the DDEEC_E is the third, and lastly, the DEEC presented the lowest number of first nodes dead.

Figure 5 presents the results of the first node dead (FND) at $0.8 \mathrm{~J}$. Figure 5 shows that the novel DEEC-Gauss algorithm outperformed other state-of-the-art approaches for the first node dead (FND) at $0.8 \mathrm{~J}$ initial energy. The number of first nodes dead for DEEC-Gauss has the highest stability followed by, DEEC_E, DDEEC_E, and DEEC that has the lower number of first nodes dead from the figure.

Hence, we can conclude that our proposed method aids in a longer stability period of the WSN. We use 0.1 Popt parameters that were generated randomly. The proposed DEEC-Gauss has a great privilege in delaying the round of iterations by elongating the dead of the first node.

4.1.2. Tenth Node Dead. The tenth node dead (TND) is a crucial metric for different applications for the critique from the sensor must be stabilized to prolong the time similarity before the death of the tenth node.

Figure 6 presents the results for initial energy of $0.5 \mathrm{~J}$; it shows the performance of DEEC-Gauss clustering algorithms in terms of tenth node dead (TND) for initial energy of $0.5 \mathrm{~J}$ at 1000 to 1500 rounds. Figure 6 presents that DEECGauss outperforms another state-of-the-art algorithm

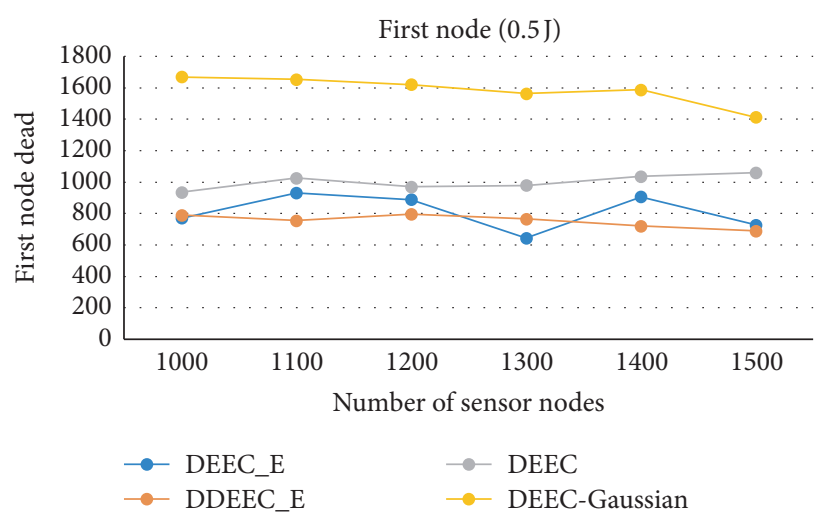

FIgURE 2: FND per 5,000 rounds with $0.5 \mathrm{~J}$.

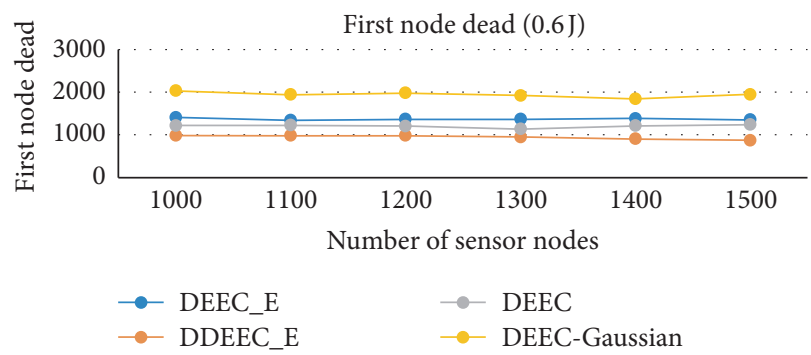

Figure 3: FND per 5,000 rounds with 0.6 J.

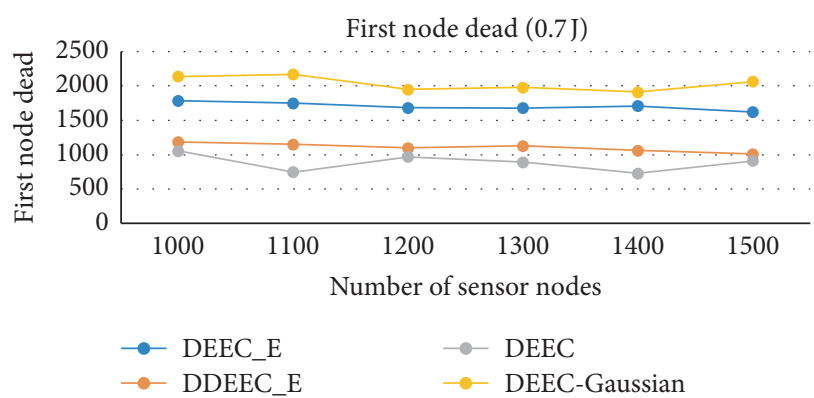

Figure 4: FND per 5,000 rounds with $0.7 \mathrm{~J}$.

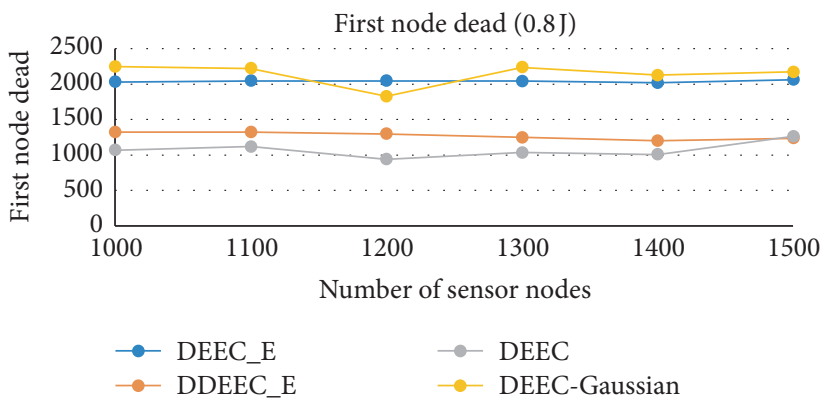

FIGURE 5: FND per 5,000 rounds with $0.8 \mathrm{~J}$.

followed by DEEC which is the second; thirdly, DEEC_E has the $3^{\text {rd }}$ highest number of stabilities followed by DDEEC_E which is the fourth.

Figure 7 presents the results for initial energy of $0.6 \mathrm{~J}$ and shows the performance of DEEC-Gauss clustering 


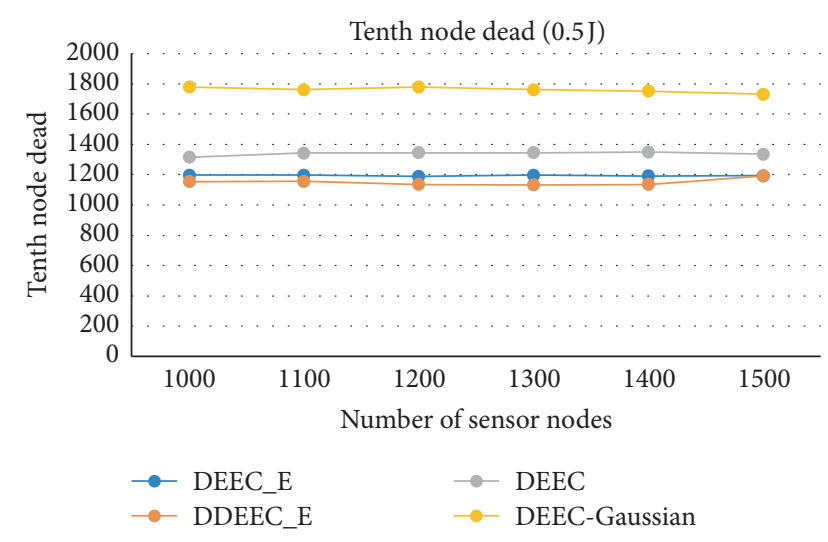

FIGURE 6: TND per 5,000 rounds with $0.5 \mathrm{~J}$.

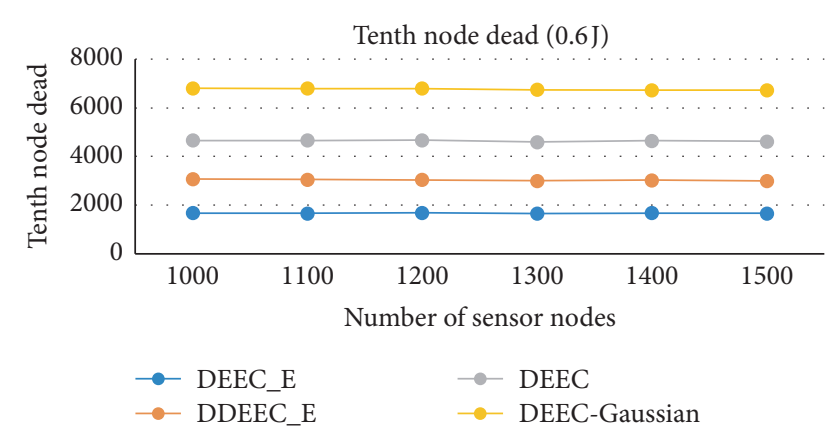

FIGURE 7: TND per 5,000 rounds with $0.6 \mathrm{~J}$.

algorithms in terms of tenth node dead (TND) for initial energy of $0.6 \mathrm{~J}$ at 1000 to 1500 rounds. It presented that DEEC-Gauss stability for the TND outperforms the other state-of-the-art algorithms followed by DEEC which is the second; thirdly, DDEEC_E has the $3^{\text {rd }}$ highest number of stabilities followed by DEEC_E which is the last.

Figure 8 presents the initial energy of $0.7 \mathrm{~J}$ at 1000 to 1500 rounds and shows the performance of the proposed DEEC-Gauss clustering algorithms in terms of tenth node dead (TND) from 1000 to 1500 rounds. Figure 8 shows that DEEC-Gauss outperforms other state-of-the-art algorithms followed by DEEC_E which is the second; thirdly, DEEC_ has the $3^{\text {rd }}$ highest number of stabilities in terms of TND, followed by DDEEC_E which is the fourth.

Figure 9 shows the performance of DEEC-Gauss clustering algorithms in terms of tenth node dead (TND) for initial energy of $0.8 \mathrm{~J}$ at 1000 to 1500 round. The figure shows that DEEC-Gauss outpaced the other state-of-the-art algorithm followed by DEEC_E which is the second; thirdly, DDEC_E has the $3^{\text {rd }}$ highest stability followed by DEEC which is the fourth. Once the individual node runs out of its energy usage, it is classified to be dead and it can no longer transmit or acknowledge any data. Lofty energy efficiency translates to low energy consumption and DEEC-Gauss aids a longer stability period compared to the state-of-the-art algorithms.

In conclusion, the result of the proposed DEEC-Gauss from Figures 2-9 was able to establish a steady duration

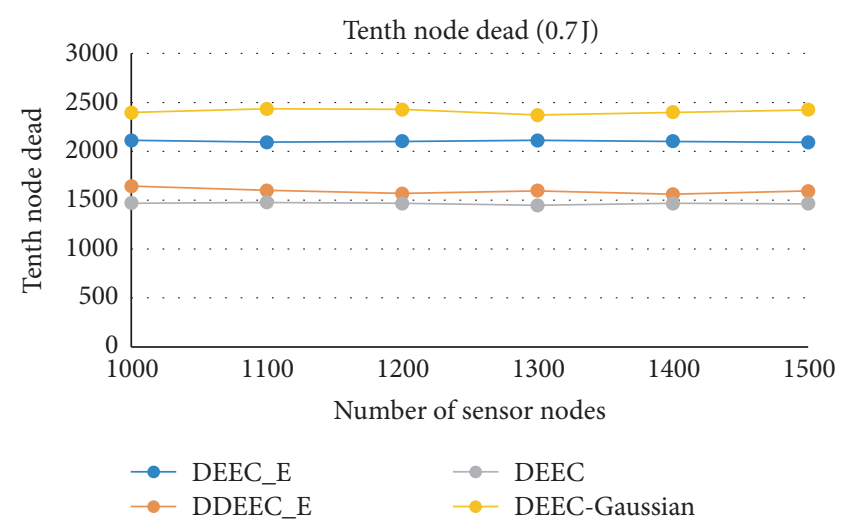

FIgURE 8: TND per 5,000 rounds with $0.7 \mathrm{~J}$.

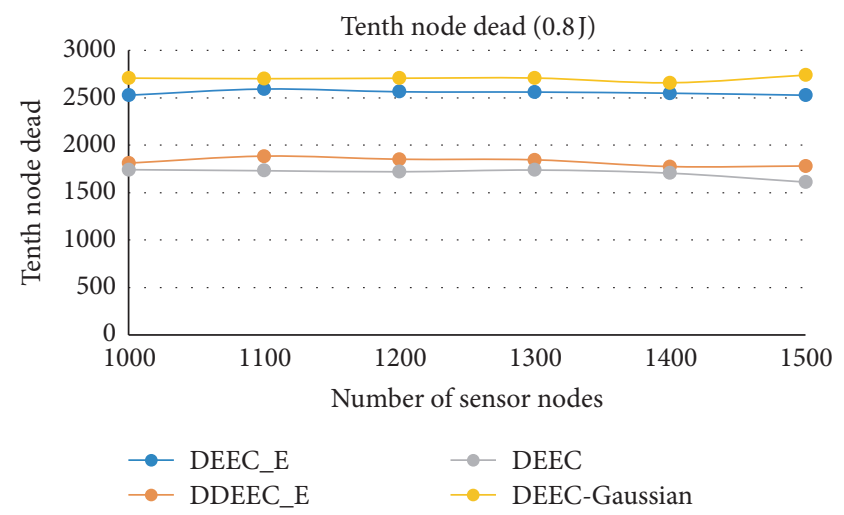

Figure 9: TND per 5,000 rounds with $0.8 \mathrm{~J}$.

before it throws up the first dead node and tenth node dead where the lifetime of the network performance tends to reduce. Therefore, taking all the simulation results into consideration, it can be deduced that FND and TND of DEEC_E, DDEEC_E, and DEEC tend to be inferior to the proposed DEEC-Gauss.

4.2. Network Throughput (Package Sent to BS for 1000 to 1500 Sensor Nodes at 5,000 Rounds). In Table 2, we observed that the number of pieces of information sent to BS from DEEC_E, DDEEC_E, DEEC, and DEEC-Gauss was computed in terms of 5,000 rounds for larger sensor nodes ranging from 1000 to 1500 sensors. Table 2 presents the average results of the packets sent to BS for initial energy of $0.5 \mathrm{~J}$; DEEC-Gauss gave the 2266873 packets, followed by DDEEC_E which is 2101192 as the second; thirdly, DEEC shows the average of 2061487 while DEEC_E average is 1962210. We observed that DEEC-Gauss outclasses other compared state-of-the-art methods.

In Table 3, we observed that the number of packages sent to BS from DEEC_E, DDEEC_E, DEEC, and DEEC-Gauss was computed in terms of 5,000 rounds for multiple sensor nodes ranging from 1000 to 1500 sensors. Table 3 presents the average results of the packets sent to BS for initial energy at 0.6 J; DEEC-Gauss evidenced 2709669 number of packets, followed by DDEEC_E which is 251992 as the second; 
TABLE 2: The packets were sent to the base station (BS) at 5,000 rounds during the network lifetime for $1000-1500$ nodes using the initial energy at $0.5 \mathrm{~J}$.

\begin{tabular}{|c|c|c|c|c|c|c|}
\hline$(0.5 \mathrm{~J})$ packets to $\mathrm{BS}$ & 1000 & 1100 & 1200 & 1300 & 1400 & 1500 \\
\hline DEEC_E & 2299147 & 1747222 & 1812327 & 1897495 & 2972858 & 1044212 \\
\hline DDEEC_E & 1651657 & 1831408 & 2011056 & 2191231 & 2359665 & 2562135 \\
\hline DEEC & 1642586 & 1814549 & 1972937 & 2141413 & 2314287 & 2483149 \\
\hline DEEC-Gaussian & 2664820 & 1969365 & 1992174 & 2142015 & 2389161 & 2443705 \\
\hline
\end{tabular}

TABLE 3: The packet was sent to the base station (BS) at 5,000 rounds during the network lifetime for $1000-1500$ nodes using the initial energy at $0.6 \mathrm{~J}$.

\begin{tabular}{|c|c|c|c|c|c|c|}
\hline$(0.6 \mathrm{~J})$ packets to $\mathrm{BS}$ & 1000 & 1100 & 1200 & 1300 & 1400 & 1500 \\
\hline DEEC_E & 2883808 & 2176074 & 1621066 & 1677306 & 2732089 & 2794223 \\
\hline DDEEC_E & 2006226 & 2203700 & 2386415 & 2615344 & 2828253 & 3080011 \\
\hline DEEC & 1933457 & 2187199 & 2370779 & 2547407 & 2755169 & 2963779 \\
\hline DEEC-Gaussian & 3038200 & 2218011 & 2422230 & 2644551 & 2920793 & 3014229 \\
\hline
\end{tabular}

TABLE 4: The packet was sent to the base station (BS) at 5,000 rounds during the network lifetime for $1000-1500$ nodes using the initial energy at $0.7 \mathrm{~J}$.

\begin{tabular}{lccccc}
\hline (0.7 J) packets to BS & 1000 & 1100 & 1200 & 1300 & 1400 \\
\hline DEEC_E & 3338721 & 390056 & 2439559 & 380501 & 2508438 \\
DDEEC_E & 2362573 & 245102 & 2800331 & 3044108 & 3306804 \\
DEEC & 2246848 & 2499249 & 2725818 & 2966581 & 3216831 \\
DEEC-Gaussian & 2247434 & 3653446 & 2822387 & 3970148 & 3450988 \\
\hline
\end{tabular}

thirdly, DEEC shows the average of 2459632 while DEEC_E average is 2314094. We observed that DEEC-Gauss outclasses other state-of-the-art algorithms.

In Table 4, we observed that the number of packets sent to BS from DEEC_E, DDEEC_E, DEEC, and DEEC-Gauss was computed in terms of 5,000 rounds for many sensor nodes ranging from 1000 to 1500 sensors. Table 4 presents the average results of the packets sent to BS for initial energy of 0.7 J; DEEC-Gauss gave the 3293861 packets, followed by DEEC which is 2850419 as the second; thirdly, DDEEC_E shows the average of 2554528 while DEEC_E average is 2101554. We observed that DEEC-Gauss has the best result compared to other state-of-the-art algorithms.

In Table 5, we observe that the number of pieces of information sent to BS from DEEC_E, DDEEC_E, DEEC, and DEEC-Gauss was computed in terms of 5,000 rounds for many sensor nodes ranging from 1000 to 1500 sensors. Table 5 presents the average results of the packets sent to BS for initial energy of $0.8 \mathrm{~J}$; DEEC-Gauss gave the 3429624 packets, followed by DDEEC_E which is 2264099 as the second; thirdly, DEEC shows the average of 2999752 while DEEC_E average is 1375729 . We observed that DEEC-Gauss surpasses other state-of-the-art methodologies.

In summary, from the result shown in Tables $2-5$, it is greatly evidenced that the proposed novel method, DEECGauss, outperformed other modish approaches in terms of information sent to BS for initial energy ranging from $0.5 \mathrm{~J}$ to $0.8 \mathrm{~J}$. The proposed DEEC-Gauss has more packets sent successfully within a short time frame with an outshine number of packets to BS which helps to minimize energy reduction.

4.3. Network Execution Lifetime for Various Algorithms. Figure 10 shows the energy execution time of sensor nodes ranging from 1000 to 1500 nodes for initial energy of $0.5 \mathrm{~J}$ as displayed above; it shows that the novel DEEC-Gauss algorithm takes minimal overall processing time, followed by DEEC_E, DDEEC_E, and DEEC.

Figure 11 displays the energy execution time of sensor nodes ranging from 1000 to 1500 nodes for initial energy of $0.6 \mathrm{~J}$ as displayed; it shows that the novel DEEC-Gauss algorithm takes the minutest processing time to outdo other state-of-the-art algorithms, followed by DDEEC_E, DEEC_E, and DEEC in terms of the network processing time.

Figure 12 evidenced the energy execution time of sensor nodes ranging from 1000 to 1500 nodes for initial energy of $0.7 \mathrm{~J}$ as displayed; it shows that the proposed DEEC-Gauss algorithm takes the least overall processing time to defeat ingenious algorithms, followed by DDEEC_E, DEEC_E, and DEEC in terms of the network processing execution time.

Figure 13 displays the energy execution time of sensor nodes ranging from 1000 to 1500 nodes for initial energy for $0.8 \mathrm{~J}$ as displayed; it shows that the novel DEEC-Gauss algorithm takes the tiniest overall processing execution time to outshine other state-of-the-art algorithms followed by 
TABLE 5: A packet was sent to the base station (BS) at 5,000 rounds during the network lifetime for 1000-1500 nodes using the initial energy at $0.8 \mathrm{~J}$.

\begin{tabular}{|c|c|c|c|c|c|c|}
\hline$(0.8 \mathrm{~J})$ packets to $\mathrm{BS}$ & 1000 & 1100 & 1200 & 1300 & 1400 & 1500 \\
\hline DEEC_E & 3723029 & 249673 & 281766 & 312635 & 331763 & 3355506 \\
\hline DDEEC_E & 2690078 & 2926016 & 3274822 & 3510145 & 3781593 & 4001940 \\
\hline DEEC & 2472324 & 2718987 & 3052043 & 3270663 & 3528774 & 2955721 \\
\hline DEEC-Gaussian & 2543934 & 2798712 & 3118411 & 3542941 & 3620260 & 4953488 \\
\hline
\end{tabular}

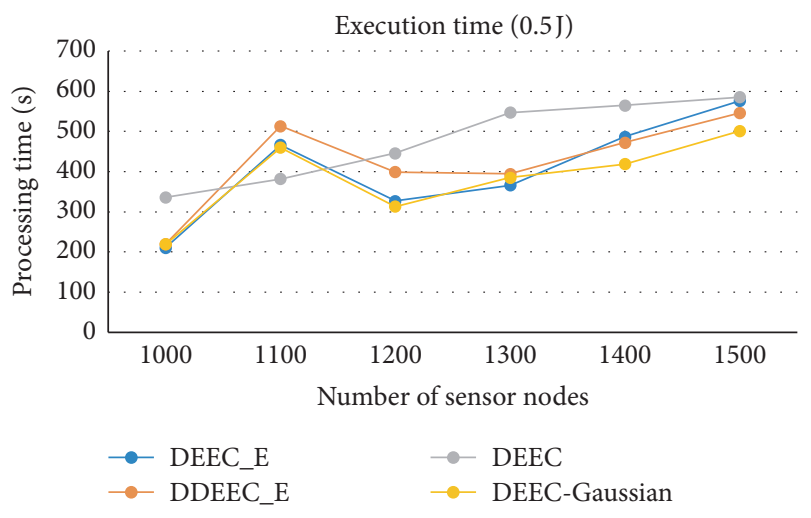

Figure 10: Execution time with $0.5 \mathrm{~J}$.

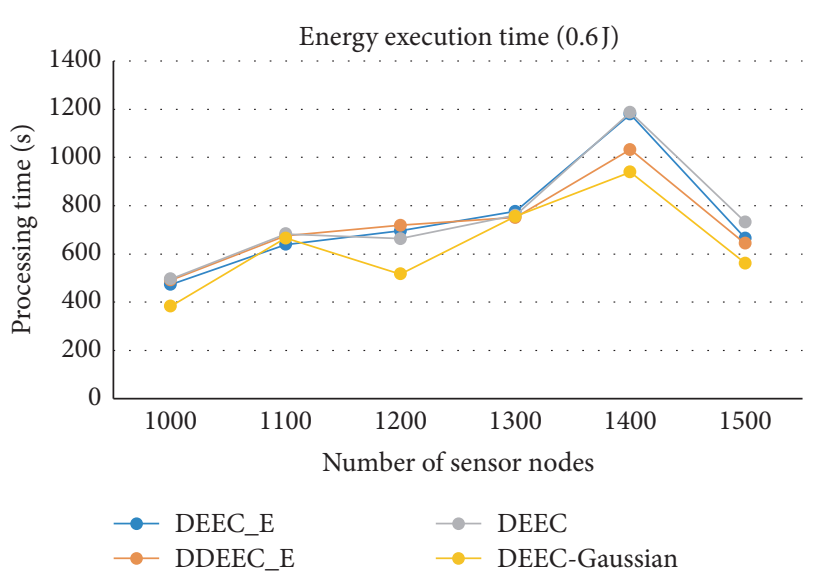

Figure 11: Execution time with $0.6 \mathrm{~J}$.

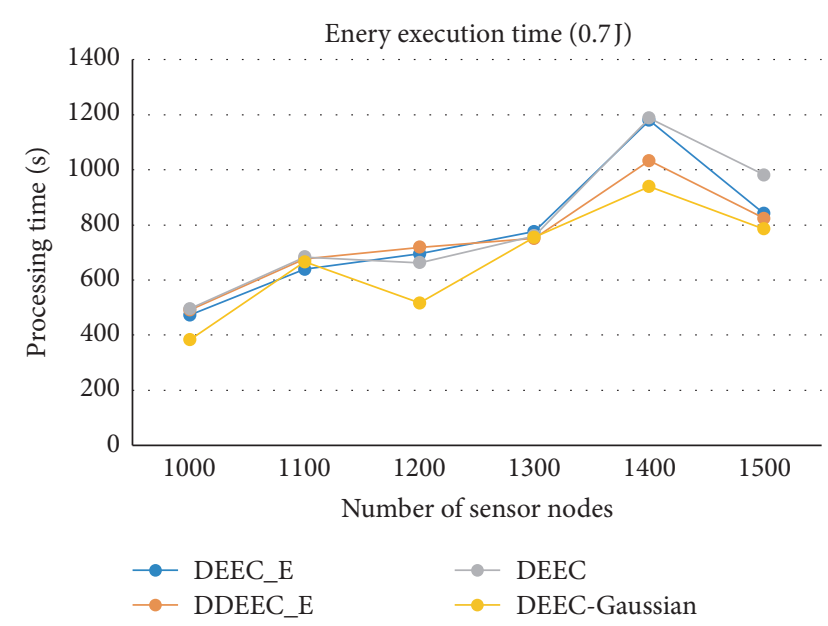

Figure 12: Execution time with $0.7 \mathrm{~J}$.

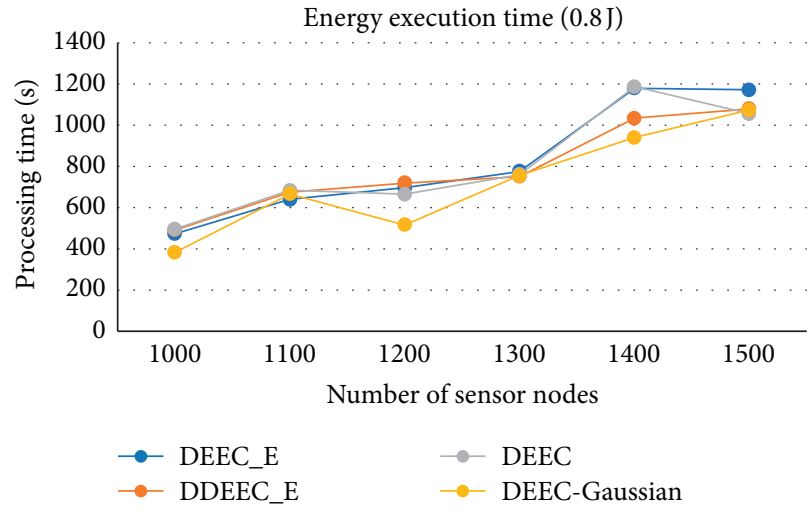

Figure 13: Execution time with $0.8 \mathrm{~J}$.

DDEEC_E, DEEC, and DEEC_E in terms of the network processing time.

Therefore, drawing inferences from all figures, we can state that the network lifetime to send successful packets to the BS for the proposed novel DEEC-Gauss algorithm was the most efficient clustering algorithm while DEEC falls into the second; DEEC_E is the third class, and DDEEC_E is the last from the simulation analysis that was performed concerning the network execution time. DEEC-Gauss spent the minimal flow to send a huge amount of information within a short amount of period for energy levels of $0.5 \mathrm{~J}$ to $0.8 \mathrm{~J}$, respectively, for all processes of nodes from 1000 to 1500 with static 5000 rounds throughout the simulation.

\section{Conclusions}

This research paper presented a hyper-heuristic multisensor energy-efficient model for the optimization of energy efficiency in wireless sensor networks for 1000 to 1500 nodes as compared to wireless sensor networks on a smaller scale [56]. The simulation result is evidenced with the presented approach of DEEC-Gauss compared with the indigenous benchmarked clustering methodology. The power efficiency management adds heterogeneity in the network by introducing minimal cluster head packets to conserve energy optimization than the state-of-the-art clustering algorithms, namely, DEEC_E, DDEEC_E, and DEEC. To sum up, the simulation shows that the presented method has the bestoptimized achievement, and it helps to increase the network lifetime and network throughput execution time to the base station, hence optimizing efficiency and enhancing the stability of the networks. This means that DEEC-Gauss overcomes more depth to overcome the challenges 
encountered by the state-of-the-art algorithms. The presented approach has an excellent duration to send packages successfully to the base station (BS) for thousands of sensor nodes. Finally, the presented approach presented an optimized energy-efficient clustering to ensure a more stabilized period for larger WSNs' flow of operation. Our recommendation for the future is to implement node localization algorithms to thousands of sensor nodes with varieties of energy requirements to evaluate their efficiency in others to overcome the challenges of node localization to pull through it for a longer duration and to add more value for the network services.

\section{Data Availability}

The data and MATLAB code for the simulations are available from the authors on request.

\section{Conflicts of Interest}

The authors declare no conflicts of interest.

\section{Acknowledgments}

This research was funded by the ICT and Society Research Group, Information Systems, Durban University of Technology.

\section{References}

[1] A. I. Ferreiro, M. Rabaçal, and M. Costa, "A combined genetic algorithm and least squares fitting procedure for the estimation of the kinetic parameters of the pyrolysis of agricultural residues," Energy Conversion and Management, vol. 125, pp. 290-300, 2016.

[2] M. Eftekhari, E. Kranakis, D. Krizanc et al., "Distributed algorithms for barrier coverage using relocatable sensors," Distributed Computing, vol. 29, no. 5, pp. 361-376, 2016.

[3] C. Omar, A. Koubaa, and A. Zarrad, "A cloud based disaster management system," Journal of Sensor and Actuator Networks, vol. 9, no. 1, p. 6, 2020.

[4] O. J. Aroba, N. Naicker, and T. Adeliyi, "Meta-analysis of heuristic approaches for optimizing node localization and energy efficiency in wireless sensor networks," International Journal of Engineering and Advanced Technology (IJEAT), vol. 10, no. 1, pp. 81-88, 2020.

[5] C. Omar and A. Koubaa, "Blockloc: secure localization in the internet of things using blockchain," in Proceedings of the 15th International Wireless Communications \& Mobile Computing Conference (IWCMC), pp. 629-634, IEEE, Tangier, Morocco, June 2019.

[6] M. Singh and P. M. Khilar, "A range free geometric technique for localization of wireless sensor network (WSN) based on controlled communication range," Wireless Personal Communications, vol. 94, no. 3, pp. 1359-1385, 2017.

[7] S. Messous and H. Liouane, "Online sequential DV-hop localization algorithm for wireless sensor networks," Mobile Information Systems, vol. 2020, Article ID 8195309, 14 pages, 2020.

[8] M. S. Mozamir, R. B. Abu Bakar, W. I. S. Wan Din, and Z. Musa, "GbLN-PSO algorithm for indoor localization in wireless sensor network," IOP Conference Series: Materials
Science and Engineering, vol. 769, no. 1, Article ID 012033, 2020.

[9] A. Prakash, R. Mishra, and A. S. Kushwaha, "Exterior bound allotted advanced node distribution for energy efficient clustering (EBAN DEEC) protocol in WSN," International Journal of Research and Development in Applied Science and Engineering (IJRDASE), vol. 20, no. 1, 2020.

[10] Moorthi and R. Thiagarajan, "Energy consumption and network connectivity based on novel-LEACH-POS protocol networks," Computer Communications, vol. 149, pp. 90-98, 2020.

[11] A. M. Bongale, C. R. Nirmala, and A. M. Bongale, "Hybrid cluster head election for WSN based on firefly and harmony search algorithms," Wireless Personal Communications, vol. 106, no. 2, pp. 275-306, 2019.

[12] N. Moussa, Z. Hamidi-Alaoui, and A. E. B. El Alaoui, "ECRP: an energy-aware cluster-based routing protocol for wireless sensor networks," Wireless Networks, vol. 26, no. 4, pp. 2915-2928, 2020.

[13] P. Rawat, S. Chauhan, and R. Priyadarshi, "Energy-efficient clusterhead selection scheme in heterogeneous wireless sensor network," Journal of Circuits, Systems, and Computers, vol. 29, no. 13, Article ID 2050204, 2020.

[14] W. Osamy, A. Salim, and A. M. Khedr, "An information entropy based-clustering algorithm for heterogeneous wireless sensor networks," Wireless Networks, vol. 26, no. 3, pp. 1869-1886, 2020.

[15] Y. Meng, Y. Zhan, J. Gu et al., "3-D localization in WSNs using UAVs," World Scientific Research Journal, vol. 6, no. 10, pp. 321-332, 2020.

[16] B. Elbhiri, R. Saadane, S. El fldhi, and D. Aboutajdine, "Developed distributed energy-efficient clustering (DDEEC) for heterogeneous wireless sensor networks," in Proceedings of the 5th International Symposium on I/V Communications and Mobile Network (ISVC), Rabat, Morocco, September 2010.

[17] Q. Li, Q. Zhu, and M. Wang, Design of a Distributed EnergyEfficient Clustering (DEEC) Algorithm for Heterogeneous Wireless Sensor Networks, School of Computer Science and Engineering, University of Electronic, Science and Technology of China, Chengdu, China, 2020.

[18] R. Anurag and Sharma, "Load forecasting by using ANFIS," International Journal of Research and Development. in Applied Science and Engineering, vol. 20, no. 1, 2020.

[19] R. K. Poluru, M. P. K. Reddy, S. M. Basha, R. Patan, and S. Kallam, "Enhanced adaptive distributed energy-efficient clustering (EADEEC) for wireless sensor networks," Recent Advances in Computer Science and Communications, vol. 13, no. 2, pp. 168-172, 2020.

[20] V. Nehra, A. K. Sharma, and R. K. Tripathi, "I-DEEC: improved DEEC for blanket coverage in heterogeneous wireless sensor networks," Journal of Ambient Intelligence and Humanized Computing, vol. 11, no. 9, pp. 3687-3698, 2020.

[21] R. R. Priyadarshini and N. Sivakumar, "Enhancing coverage and connectivity using energy prediction method in underwater acoustic WSN," Journal of Ambient Intelligence and Humanized Computing, vol. 11, no. 7, pp. 2751-2760, 2019.

[22] X. Zhao, X. Xiong, Z. Sun, X. Zhang, and Z. Sun, “An immune clone selection based power control strategy for alleviating energy hole problems in wireless sensor networks," Journal of Ambient Intelligence and Humanized Computing, vol. 11, no. 6, pp. 2505-2518, 2019.

[23] S. Singh, "A proficient node deployment mechanism using adjustable sensing range in wireless sensor networks," Iranian 
Journal of Science and Technology, Transactions of Electrical Engineering, vol. 43, no. 1, pp. 191-199, 2019.

[24] V. Srivastava, S. Tripathi, and K. Singh, "Energy efficient optimized rate based congestion control routing in wireless sensor network," Journal of Ambient Intelligence and $\mathrm{Hu}$ manized Computing, vol. 11, no. 3, pp. 1325-1338, 2019.

[25] D. Anuradha and S. K. Srivatsa, "Energy effectual reconfigurable routing protocol (E2R2P) for cluster based underwater wireless sensor networks," Journal of Ambient Intelligence and Humanized Computing, pp. 1-8, 2019.

[26] J. Bhola, S. Soni, and G. K. Cheema, "Genetic algorithm based optimized leach protocol for energy efficient wireless sensor networks," Journal of Ambient Intelligence and Humanized Computing, vol. 11, no. 3, pp. 1281-1288, 2019.

[27] P. Gupta and A. K. Sharma, "Designing of energy efficient stable clustering protocols based on BFOA for WSNS," Journal of Ambient Intelligence and Humanized Computing, vol. 10, no. 2, pp. 681-700, 2019.

[28] F. Sharma, M. I. Daabo, and K. A. Gbolagade, "Gateway-stable election protocol for heterogeneous wireless sensor network," Asian Journal of Research in Computer Science, vol. 5, no. 1, pp. 40-48, 2020.

[29] F. Jibreel, "Improved enhanced distributed scheme for heterogeneous wireless sensor network," International Journal of Engineering Research and Advanced Technology (IJERAT), vol. 5, no. 1, pp. 6-11, 2019.

[30] K. Kaur and E. S. Sharma, "Analysis grid based DEEC protocol with priority queue for increasing lifetime of WSN," IJRAR-International Journal of Research and Analytical Reviews (IJRAR), vol. 7, no. 2, pp. 699-703, 2020.

[31] V. Dhiman, M. Kumar, M. Kumar, and A. K. Sharma, "A radical study of energy efficient hierarchical cluster-based routing protocols for WSN," International Journal on Electrical Engineering and Informatics, vol. 12, no. 3, pp. 445-469, 2020.

[32] L. Kumar and D. Patle, "Lifetime enhancement in wireless sensor networks using adaptive clustering and thresholding," International Journal of Innovative Research in Echnology and Management, vol. 4, no. 1, 2020.

[33] H. Mohapatra, A. Rath, P. B. Landge et al., "A comparative analysis of clustering protocols of wireless sensor network," International Journal of Mechanical and Production Engineering Research and Development (IJMPERD), vol. 9, no. 3, pp. 2249-6890, 2020.

[34] M. Mehrani, J. Shanbezadeh, A. Sarrafzadeh et al., "Feed: fault tolerant, energy efficient, distributed clustering for WSN," in Proceedings of the 12th International Conference on Advanced Communication Technology (ICACT), Phoenix Park, Korea, February 2010.

[35] H. Mohapatra, "A tutorial on PowerShell pipeline and its loopholes," International Journal of Emerging Trends in Engineering Research, vol. 8, no. 4, pp. 975-982, 2020.

[36] H. Mohapatra, "Handling of man-in-the-middle attack in WSN through intrusion detection system," International Journal of Emerging Trends in Engineering Research, vol. 8, no. 5, pp. 1503-1510, 2020.

[37] R. Kumar, S. Jha, and R. Singh, "A different approach for solving the shortest path problem under mixed fuzzy environment," International Journal of Fuzzy System Applications, vol. 9, no. 2, pp. 132-161, 2020.

[38] R. Kumar, S. A. Edalatpanah, S. Jha, and R. Singh, “A novel approach to solve Gaussian valued neutrosophic shortest path problems," International Journal of Engineering and Advanced Technology, vol. 8, 2019.
[39] S. Gayen, F. Smarandache, S. Jha et al., "Introduction to plithogenic hypersoft subgroup," Neutrosophic Sets and Systems, vol. 33, no. 1, 2020.

[40] R. Kumar, S. A. Edalatpanah, and H. Mohapatra, "A note on optimal path selection approach for fuzzy reliable shortest path problem," Journal of Intelligent \& Fuzzy Systems, vol. 32, no. 1, pp. 1-4, 2020.

[41] S. V. Purkar and R. S. Deshpande, "Clustering algorithm for deployment of independent heterogeneous wireless sensor network," Wireless Personal Communications, vol. 112, pp. 1-15, 2020.

[42] A. Rahiminasab, P. Tirandazi, M. J. Ebadi, A. Ahmadian, and M. Salimi, "An energy-aware method for selecting cluster heads in wireless sensor networks," Applied Sciences, vol. 10, no. 21, p. 7886, 2020.

[43] T. A. Alghamdi, "Energy efficient protocol in wireless sensor network: optimized cluster head selection model," Telecommunication Systems, vol. 74, no. 3, pp. 331-345, 2020.

[44] C. Ju, Y. Gao, A. K. Sangaiah, and G. J. Kim, "A PSO based energy efficient coverage control algorithm for wireless sensor networks," Computers, Materials \& Continua, vol. 56, no. 3, pp. 433-446, 2018.

[45] D. Gao, S. Zhang, F. Zhang, X. Fan, and J. Zhang, "Maximum data generation rate routing protocol based on data flow controlling technology for rechargeable wireless sensor networks," Computers, Materials \& Continua, vol. 59, no. 2, pp. 649-667, 2019.

[46] A. Janarthanan and D. Kumar, "Localization based evolutionary routing (LOBER) for efficient aggregation in wireless multimedia sensor networks," Computers, Materials \& Continua, vol. 60, no. 3, pp. 895-912, 2019.

[47] J. Wang, Y. Gao, X. Yin, F. Li, and H. J. Kim, “An enhanced PEGASIS algorithm with mobile sink support for wireless sensor networks," Wireless Communications and Mobile Computing, vol. 2018, Article ID 9472075, 9 pages, 2018.

[48] J. Wang, X. Gu, W. Liu, A. K. Sangaiah, and H. J. Kim, “An empower Hamilton loop based data collection algorithm with mobile agent for WSNs," Human-centric Computing and Information Sciences, vol. 9, no. 1, pp. 1-14, 2019.

[49] J. Wang, Y. Gao, C. Zhou, R. Simon Sherratt, and L. Wang, "Optimal coverage multi-path scheduling scheme with multiple mobile sinks for WSNs," Computers, Materials \& Continua, vol. 62, no. 2, pp. 695-711, 2020.

[50] H. Mostafaei and M. S. Obaidat, "May. A distributed efficient algorithm for self-protection of wireless sensor networks," in Proceedings of the 2018 IEEE International Conference on Communications (ICC), pp. 1-6, IEEE, Kansas City, MO, USA, May 2018.

[51] H. Mostafaei, M. U. Chowdhury, R. Islam, and H. Gholizadeh, "Connected P-Percent coverage in wireless sensor networks based on degree constraint dominating set approach," in Proceedings of the 18th ACM International Conference on Modeling, Analysis and Simulation of Wireless and Mobile Systems, pp. 157-160, Cancun, Mexico, November 2015.

[52] T. A. Al-Janabi and H. S. Al-Raweshidy, "Optimised clustering algorithm-based centralised architecture for load balancing in IoT network," in Proceedings of the 2017 International Symposium on Wireless Communication Systems (ISWCS), pp. 269-274, IEEE, Bologna, Italy, August 2017.

[53] W. B. Heinzelman, A. P. Chandrakasan, and H. Balakrishnan, "An application-specific protocol architecture for wireless microsensor networks," IEEE Transactions on Wireless Communications, vol. 1, no. 4, pp. 660-670, 2002. 
[54] S. Gharib, S. R. Ali, R. Khan, and N. Munir, "System of linear equations, Guassian elimination," Global Journal of Computer Science and Technology, vol. 15, no. 5, 2015.

[55] L. d. O. Miranda and L. B. B. Miranda, "Lohans' magic squares and the Gaussian elimination method," Journal of Nepal Mathematical Society, vol. 3, no. 1, pp. 31-36, 2020. 